\title{
Antimicrobial Resistance Pattern of Bacterial Meningitis Among Patients in Quetta, Pakistan
}

\author{
Syeda Ayesha Ali $\mathbb{D}^{1,2}$ \\ Muhammad Kamran Taj ${ }^{1}$ \\ Syeda Hafsa $\mathrm{Ali}^{3}$ \\ 'Center for Advanced Studies in \\ Vaccinology and Biotechnology \\ (CASVAB), University of Balochistan, \\ Quetta, Pakistan; ${ }^{2}$ Department of \\ Biochemistry, Sardar Bahadur Khan \\ Women's University (SBKWU), Quetta, \\ Pakistan; ${ }^{3}$ Department of Microbiology, \\ Balochistan University of Information \\ Technology, Engineering, and \\ Management Sciences (BUITEMS), \\ Quetta, Pakistan
}

\begin{abstract}
Introduction: Bacterial meningitis $(\mathrm{BM})$ is a lethal infectious disease that persists as a substantial cause of morbidity and mortality. The antimicrobial drug resistance in BM pathogens poses a major threat to the community while raising challenges for clinicians. For the first time, we elucidate the incidence rate of BM and its drug resistance among the population of Quetta, Balochistan.
\end{abstract}

Methods: A cross-sectional study was conducted (from January 2018 to March 2021) among meningitis patients admitted to government hospitals in Quetta. The cerebrospinal fluid (CSF) specimen was collected and processed for microbiological and cytological analysis. Bacterial isolates were identified and confirmed using phylogenetic analysis of the 16S rRNA gene.

Results: We found 35.9\% (321/894) confirmed cases of BM among the population of Quetta, Balochistan. The identified bacterial isolates comprised Streptococcus pneumoniae 14.7\% (130/894), Staphylococcus aureus 6.9\% (61/894), Neisseria meningitidis 5.7\% (51/ 894), Haemophilus influenzae 2.5\% (22/894), Escherichia coli 4.5\% (40/894), and Klebsiella pneumoniae $1.9 \%$ (17/894). A high percentage in male patients of $20.8 \%(186 / 894)$ was identified as compared to female patients of $15.1 \%$ (135/894). Extreme age groups such as infants (age range: 1 month to 1 year) and adults (age range: 61 years to 99 years) with low immunity were critically affected by BM. Clinical parameters such as abnormal CSF appearance, $\mathrm{pH}$, and high WBCs in $\mathrm{BM}$ patients with endocarditis as the underlying disease were significantly at elevated risk of fatal outcome. 16S rRNA gene phylogenetic analysis confirmed the evolutionary similarity of isolated strains with prevailing pathogenic strains in Asia. Furthermore, age was identified as a significant risk factor for clinical outcomes.

Conclusion: The majority of the identified BM patients belonged to rural areas with limited health-care facilities and paramedic staff. The high case fatality rate $11 \%$ demonstrates increased antibiotic resistance among BM isolates, thereby stimulating its devastation in underdeveloped regions. Timely detection and intensive treatment of BM may improve critical outcomes in patients.

Keywords: bacterial meningitis, cerebrospinal fluid, 16S rRNA, incidence rate, drug resistance

\section{Introduction}

Bacterial meningitis (BM) is a serious global health challenge. BM involves inflammation of the meninges that protect the human brain and spinal cord. Bacterial infection, if left untreated, progresses to bacteremia and evades the immune system. It eventually infiltrates and disrupts the blood-brain barrier (BBB) and the nervous system. ${ }^{1}$ The classical signs such as fever, headache and neck stiffness were frequently observed among BM patients. ${ }^{2,3}$ The commonly
Correspondence: Muhammad Kamran Taj Center for Advanced Studies in Vaccinology and Biotechnology (CASVAB), University of Balochistan, Quetta, 87300, Pakistan

Email kamrancasvab@yahoo.com 
known causative agents of BM are Streptococcus pneumoniae, Haemophilus influenzae type b. Streptococcus group B, Staphylococcus aureus, Listeria monocytogenes and Neisseria meningitidis. ${ }^{1}$ The case-fatality rate of BM is high and varies over time, location, and host characteristics such as patient age, gender etc. ${ }^{4-7}$ High morbidity and mortality rates are commonly reported among underdeveloped countries. ${ }^{6}$ In untreated patients, the fatality ratio might increase by up to $70 \%$, while one out of five survivors could be afflicted with neurological disabilities. ${ }^{6,8,9}$ Early screening of pathogens with relevant treatment may prevent the case fatality rate and neurological complications. Antibiotics play an imperative role in reducing the fatality rate of infectious diseases. In spite of advancements in the healthcare system and therapeutics, the increased mortality rate is concerning. ${ }^{10}$ For a long time, penicillin, cephalosporin, fluoroquinolones, or aminoglycosides were empirical therapy to treat BM. Nevertheless, these antibiotics were ineffective against resistant $\mathrm{BM}$ isolates. ${ }^{9}$ For example, third-generation antibiotics (cephalosporin) are ineffective against Escherichia coli meningitis, as it increases mortality and morbidity rates as well as contributing to complications in 25 to $50 \%$ of survivors. ${ }^{5}$ This prompt emergence of antimicrobial resistance (AMR) has raised substantial health concerns in developing and under-developed countries. Among diagnostic tests to identify BM, bacterial culturing remains a gold standard. ${ }^{5}$ However, bacterial culturing of CSF sometimes lacks sensitivity owing to the use of antibiotics prior to lumbar puncture (LP), as well as low quantity and quality of CSF, which impedes the identification of causative agents. Molecular based identification is vital for confirmation of pathogens in biological samples. Targeting $16 \mathrm{~S}$ rRNA genes (an evolutionarily conserved region) offers prompt detection of bacteria. Furthermore, 16S rRNA associated phylogenetic analysis of unknown isolates can identify evolutionary relationships between the reported strains. Monitoring molecular epidemiology and genetic variation of pathogenic strains helps to track microbial resistance and prevent future outbreaks of infectious diseases. ${ }^{11}$ Therefore, periodic inspections are vital for evaluating fatal diseases.

Balochistan is the largest province in Pakistan with scarcity of health-care resources, impoverished population, with low socioeconomic status compared to its sister provinces. Inadequate epidemiological representation of bacterial infections and their emerging antibiotic resistance is a major challenge in Balochistan. ${ }^{12,14}$ The epidemiological data assists in making decisions in terms of therapeutic and curative approaches among BM patients. However, the data for meningitis are insufficient in Pakistan. Balochistan has inadequate resources and poses immense challenge in retrieving BM data; as a result, this disease remains neglected in the province with poor management and prognosis. Here, we for the first time explored the etiological cause of BM and its antibiotic resistance patterns among patients in Quetta City, Balochistan Province from 2018 to 2021. We aimed to evaluate clinical complications, risk factors and comorbid issues associated with $\mathrm{BM}$ in the region and its mortality rate among the patients.

\section{Materials and Methods Samples Collection}

A total of 894 patients suspected of having BM were admitted to Bolan Medical Complex Hospital (BMCH) and District Hospital Quetta (DHQ) from 1st January 2018 to 31st March 2021. Both are government hospitals with tertiary medical health-care facilities located in Quetta, the provincial capital of Balochistan, Pakistan. Oral and written consent was taken from patients and from their parents/guardians in the case of children, before collecting CSF samples. One CSF sample $(\sim 1-3 \mathrm{~mL})$ from each patient was collected via LP. Samples were collected aseptically in a sterile tube according to the guidelines of the ethical committees of hospitals and institutions. This research was conducted according to the Helsinki declaration, the University of Balochistan (CAS/ 45/15-20), and the hospital's ethical committee's permission (E.C 18-12/2017). The collected samples were immediately processed for further microbiological processing at the Center for Advanced Studies in Vaccinology and Biotechnology (CASVAB), University of Balochistan, Quetta.

\section{Patients' Demographic Data}

To identify risk factors among BM patients in Quetta, epidemiological data were collected. A questionnaire was constructed to record demographics such as gender, age group, and socioeconomic status. The following groups were included according to their salary/income in Pakistani Rupees (PKR) per month: upper class $(>50,000$ PKR), middle class (21,000-50,000 PKR) and lower class $(<20,000$ PKR). Educational status was identified as literate and illiterate. The clinical features of patients were 
recorded as high fever, unconsciousness, seizures/partial fits (if age below 5 years), and neck stiffness. ${ }^{4}$ Comprehensive examinations of all patients were done by professional doctors in an emergency, pediatrics, and neuro-medicine wards before LP.

\section{Laboratory Examination}

Extensive investigation of CSF samples included: Gram staining, culture, CSF pH, appearance, WBC count, and glucose and protein level. Gram staining and culturing of CSF specimens were immediately performed. Delay in microbiological procedures and use of antibiotics before LP might affect the sensitivity of staining and culturing. Only positive samples in culture for bacterial isolates were considered in this study. Moreover, analysis for mycobacteria, fungal, or viral pathogens was performed at the doctor's discretion. The inclusion criteria for BM were a) identification of a specific bacterial agent (from CSF) in culture media, clinical history, and inflammation of the meninges b) Acute BM clinical signs and symptoms include fever, fits, vomiting, headache, neck stiffness, and unconsciousness. c) CSF has distinct characteristics such as increased WBCs, low glucose levels, and a high protein level.

\section{Isolation and Identification}

The CSF samples were centrifuged, and the pellets containing isolates were inoculated on blood and chocolate agar plates. Inoculated plates were aerobically incubated with $5 \% \mathrm{CO}_{2}$ for approximately 48 hours at $37^{\circ} \mathrm{C}$. After the incubation period, the colonies of bacteria were identified and observed using standard methods such as gram staining and PCR identification.

\section{Sanger Sequencing and Phylogenetic Analysis of Isolates}

PCR of the 16S rRNA gene was further confirmed. The total genomic DNA of isolates was extracted via a DNA purification kit (GeneAll, Korea). The 16S rRNA gene was used for identification with primer sequences for each specific primer. These primers were used to amplify their respective PCR fragments (Table 1). The sequencing of the 16S rRNA gene of all the isolates was shipped to the Macrogen Company. The ABI genetic analysis systems used the standard forward and reverse 16S rRNA primers for sequencing of the isolates. The obtained sequences were blasted with reported sequences in the NCBI database. Furthermore, the similarities among the reference sequences were analysed in bioedit software. The sequence consensuses that had a similarity of $90 \%$ and above were included in this study. ${ }^{14}$ Multiple sequence alignment was also performed. For the phylogenetic tree, the analysis was performed in MEGA X. ${ }^{15}$ First, clustering was performed via the Neighbor-joining method. Further, the analysis of the tree was conducted by the maximum composite likelihood method. Finally, the cut-off values $\left(\geq 75 \%\right.$ ) for each node ${ }^{15}$ were determined.

\section{Antibiotic Susceptibility Test}

The antibiotic susceptibility test was performed according to the standard method recommended by the CLSI. We used 12 antibiotics such as meropenem $(10 \mu \mathrm{g})$, ceftazidime $(30 \mu \mathrm{g})$,

Table I The Primer Sequences for Diagnosis of Bacterial Isolates BM Patients

\begin{tabular}{|l|l|l|c|c|c|}
\hline S. No. & Strain & Primer I6S rRNA & bps & Tm Values & Reference \\
\hline I. & E. coli & $\begin{array}{l}\text { Forward primer= GGGAGTAAAGTTAATACCTTTGC } \\
\text { Reverse primer= CTCAAGCTTGCCAGTATCAG }\end{array}$ & 204 & 58 & 46 \\
\hline 2. & S. pneumoniae & $\begin{array}{l}\text { Forward primer= CCACACTGGGACTGAGACAC } \\
\text { Reverse primer= TCAACCTTGCGGTCGTACTC }\end{array}$ & 598 & 59 & This study \\
\hline 3. & S. aureus & $\begin{array}{l}\text { Forward primer = ACGGTCTTGCTGTCACTTATA } \\
\text { Reverse primer = TACACATATGTTCTTCCCTAATAA }\end{array}$ & 257 & 57 & 47 \\
\hline 4. & K. pneumoniae & $\begin{array}{l}\text { Forward primer= TCTGAGAGGATGACCAGCCA } \\
\text { Reverse primer= GTTTACGGCGTGGACTACCA }\end{array}$ & 525 & 59 & This study \\
\hline 5. & N. Meningiidtis & $\begin{array}{l}\text { Forward primer = TTTTGGACAATGGGCGCAAG } \\
\text { Reverse primer = GGGTCCCCGTCAATTCCTTT }\end{array}$ & 568 & 59 & This study \\
\hline 6. & H. influenzae & $\begin{array}{l}\text { Forward primer = ATACCCTGGTAGTCCACGCT } \\
\text { Reverse primer = AGTTGCAGACTCCAATCCGG }\end{array}$ & 528 & 60 & This study \\
\hline
\end{tabular}


ceftriaxone $(30 \mu \mathrm{g})$, erythromycin $(15 \mu \mathrm{g})$, amoxicillin (25 $\mu \mathrm{g})$, rifampicin $(05 \mu \mathrm{g})$, oxytetracycline $(30 \mu \mathrm{g})$, gentamicin $(10 \mu \mathrm{g})$, vancomycin $(30 \mu \mathrm{g})$, ciprofloxacin $(05 \mu \mathrm{g})$, levofloxacin $(05 \mu \mathrm{g})$ and amikacin $(30 \mu \mathrm{g})$. The opacity of the inoculums was adjusted to $0.5 \mathrm{McF}$ arland standards. The isolates were well spread over Mueller-Hinton Agar (MHA) (Oxoid Limited, England) plates whereas fastidious bacterial isolates were subcultured on 5\% sheep blood supplemented MHA plates. The antibiotic discs were placed at an equal distance on the bacterial inoculated plates. Finally, the plates were incubated for 24 hours at $37^{\circ} \mathrm{C}$ with $5 \% \mathrm{CO}_{2}$ and results were recorded $(\mathrm{mm}){ }^{16}$

\section{Statistical Analysis}

The demographics of the patients with their CSF characteristics and clinical features were analysed using multinomial regression and Pearson chi-square. The frequency of the antibiotic susceptibility test was analysed using SPSS version 20 (SPSS Inc., Chicago, IL, USA). Those values considered significant had a $\mathrm{P} \leq 0.05$.

\section{Results}

\section{Demographics of Patients}

A total of 894 patients were enrolled in this study, out of which $35.9 \%$ (321/894) of the cases were BM positive (Figure 1). The majority of BM positive cases were from BMCH, 20.6\% (184/894) and DHQ, 15.3\% (137/894). Of $321 \mathrm{BM}$ positive cases, high percentage of male patients $20.8 \%(186 / 894)$ were infected than $15.1 \%(135 / 894)$ females. BM were significantly found among children under one year old (range: 1 month-3 months; and 4 months-1 year) and in adults with age ranging between 61 and 99 years with $10.6 \%(94 / 894), 8.9 \%(80 / 894)$ and

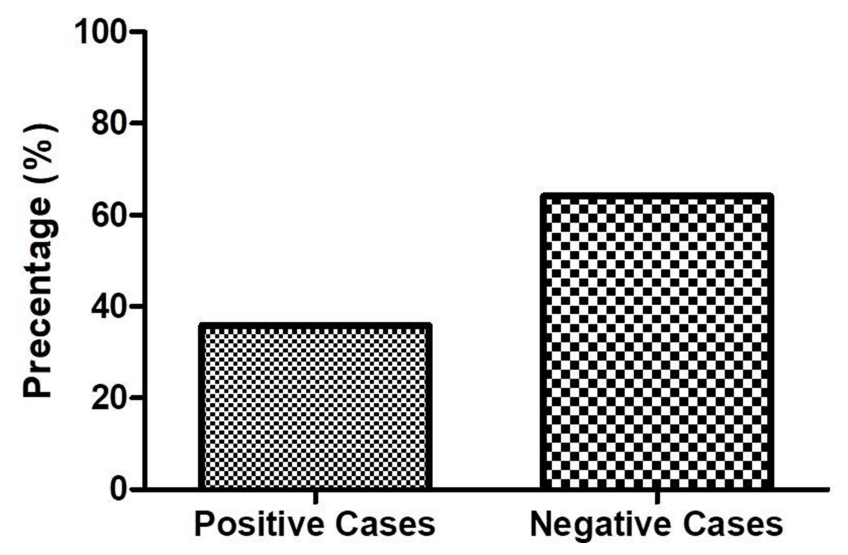

Figure I Frequency of positive bacterial meningitis cases.
$5.7 \%(51 / 894)$ frequencies, respectively (Table 2). Mostly, affected patients belonged to the Pashtun ethnic group with $19.7 \%$ (176/894). The majority of the patients belonged to the rural areas of Balochistan 18.2\% (163/ 894). The total case fatality rate was $11 \%(36 / 321)$ among the BM patients in 2018-2021 (Table 2).

Gram staining of the samples was compared to culture to identify the validity of the results (Table 3 ). The positive predictive value and negative predictive value of gram staining are $100 \%$ and $67.9 \%$, respectively. The McNemar test determined that the slight agreement between gram staining and bacterial culture $($ Kappa $=0.191)$ was significant $(\mathrm{p}<0.001)$.

The persistent bacterial isolates from CSF of various age groups were $S$. pneumoniae $14.7 \%$ (130/894), S. aureus $6.9 \%(61 / 894)$, N. meningitidis $5.7 \%$ (51/894), H. influenzae $2.5 \%$ (22/894), E. coli $4.5 \%$ (40/894), and K. pneumoniae $1.9 \%$ (17/894). In CSF of children aged less than 1 year and older adult patients $(61+$ years), S. pneumoniae, N. meningitidis, E. coli, and S. aureus were widespread while $H$. influenzae and $K$. pneumoniae were dominant among ages 13 years to 60 years old (Table 4).

\section{Clinical Parameters}

The CSF 24.4\% (218/894) of patients was collected prior to antibiotic treatment, while in $11.5 \%(103 / 894)$ of patients the CSF was collected within 24 hours of antibiotic inception (Table 5). The majority of the cases $21.3 \%$ (190/894), had significantly elevated WBC counts ranging from 101 to 2000 cells $/ \mathrm{mm}^{3}$. About $34.5 \%(308 / 894)$ patients had a raised protein level $(>45 \mathrm{mg} / \mathrm{dL})$, while $20.8 \%(186 / 894)$ patients had predominantly high neutrophil levels (range $>61-90 \%$ ) in CSF. The low glucose level predominates the cases with $27.7 \%$ (248/894), range from 0 to $44 \mathrm{mg} / \mathrm{dL}$ (Table 5). Furthermore, the frequently observed clinical features in all BM patients during the course of hospitalization were fever $35.9 \%$ $(321 / 894)$, followed by headache $30.2 \%$ (270/894), unconsciousness $18.6 \%$ (167/894), and neck stiffness $16.7 \%$ (150/894) (Figure 2).

Majority of BM afflicted patients had underlying diseases such as $18 \%(161 / 894)$ had bacteremia, $17.3 \%$ (155/ 894) with severe pneumonia, followed by chronic obstructive pulmonary disease (COPD) 11.7\% (105/894), and sinusitis $10.2 \%$ (91/894). The least comorbid diseases were diabetes mellitus $3.2 \%$ (29/894), otitis media $2.7 \%$ (24/894), and endocarditis 2.3\% (21/894) (Table 5). 
Table 2 Demographics of Bacterial Meningitis Infected Patients

\begin{tabular}{|c|c|c|c|c|}
\hline Characteristics & BM Cases n(\%) & Non-BM Cases n(\%) & Total n(\%) & p-value \\
\hline Total & 321 (35.9\%) & $573(64.1 \%)$ & $894(100 \%)$ & \\
\hline \multicolumn{5}{|c|}{ Gender } \\
\hline Male & $186(20.8 \%)$ & $342(38.3 \%)$ & $528(59.1 \%)$ & $0.6 \mathrm{II}$ \\
\hline Female & 135 (I5.1\%) & $231(25.8 \%)$ & $366(40.9 \%)$ & \\
\hline \multicolumn{5}{|c|}{ Among various age groups } \\
\hline I month- 3 months & $94(10.6 \%)$ & $42(4.7 \%)$ & $136(15.2 \%)$ & $0.00 I^{*}$ \\
\hline 4 months- I year & 80 (8.9\%) & 128 (14.3\%) & 208 (23.3\%) & \\
\hline 2 years -12 years & $46(5.1 \%)$ & $112(12.5 \%)$ & $158(17.7 \%)$ & \\
\hline 13 years -19 years & $13(1.5 \%)$ & $46(5.1 \%)$ & $59(6.6 \%)$ & \\
\hline 20 years -40 years & 17 (1.9\%) & $73(8.2 .4 \%)$ & $90(10.1 \%)$ & \\
\hline $4 I$ years -60 years & $20(2.2 \%)$ & 85 (9.5\%) & 105 (II.7\%) & \\
\hline 61 years-99 years & $5 \mathrm{I}(5.7 \%)$ & 87 (9.7\%) & $138(15.4 \%)$ & \\
\hline \multicolumn{5}{|c|}{ Resident } \\
\hline Rural & $163(18.2 \%)$ & $215(24.0 \%)$ & $378(42.3 \%)$ & $0.001 *$ \\
\hline Urban & $158(17.7 \%)$ & $358(40.0 \%)$ & $516(57.7 \%)$ & \\
\hline \multicolumn{5}{|c|}{ Ethnicity } \\
\hline Pashtun & 176 (19.7\%) & $306(34.2 \%)$ & $482(53.9 \%)$ & 0.343 \\
\hline Baloch & $68(7.6 \%)$ & $113(12.6 \%)$ & I8I (20.2\%) & \\
\hline Punjabi & $5 \mathrm{I}(5.7 \%)$ & $117(13.1 \%)$ & $168(18.8 \%)$ & \\
\hline Hazara & $26(2.9 \%)$ & 37 (4.1\%) & $63(7 \%)$ & \\
\hline \multicolumn{5}{|c|}{ Socioeconomic status } \\
\hline Lower class $<20 \mathrm{~K}$ PKR per month & 173 (19.4\%) & $243(27.2 \%)$ & $416(46.5 \%)$ & $0.003 *$ \\
\hline Middle class 2 I k-50k PKR per month & $119(13.3 \%)$ & $255(28.5 \%)$ & $374(41.8 \%)$ & \\
\hline High class $>50 \mathrm{k}$ PKR per month & $29(3.2 \%)$ & $75(8.4 \%)$ & $104(11.6 \%)$ & \\
\hline \multicolumn{5}{|c|}{ Education level } \\
\hline Illiterate & $234(26.2 \%)$ & $36 \mathrm{I}(40.5 \%)$ & $595(66.6 \%)$ & $0.003 *$ \\
\hline Literate & 87 (9.7\%) & $212(23.7 \%)$ & 299 (33.4\%) & \\
\hline \multicolumn{5}{|c|}{ Survival rate } \\
\hline Mortality & $36(4 \%)$ & $13(1.5 \%)$ & 49 (5.5\%) & $0.001 *$ \\
\hline Survive & 285 (3I.5\%) & $560(63 \%)$ & 867 (94.5\%) & \\
\hline Case fatality rate & $36(11 \%)$ & - & $36(11 \%)$ & \\
\hline \multicolumn{5}{|c|}{ Hospital admitted } \\
\hline $\mathrm{BMCH}$ & I84 (20.6\%) & $322(36.0 \%)$ & $506(56.6 \%)$ & 0.745 \\
\hline DHQ & 137 (I5.3\%) & 251 (28.1\%) & $388(43.4 \%)$ & \\
\hline
\end{tabular}

Note: *Statistically significant; $20 \mathrm{~K}=20,000 ; 50 \mathrm{k}=50,000 ; \mathrm{PKR}=$ Pakistani Rupee.

Abbreviations: BMCH, Bolan Medical Complex Hospital; DHQ, District Hospital Quetta.

The essential prognostic factors for the evaluation of fatal and non-fatal outcomes of BM diseases are listed in Table 5. The statistical analysis of variables such as CSF laboratory parameters, underlying diseases, and clinical features revealed significant prognostic factors such as
CSF pH $(P \leq 0.01)$, CSF appearance $(P \leq 0.001), \mathrm{CSF}$ WBCs $(P \leq 0.001)$, and endocarditis $(P \leq 0.05)$. Furthermore, the regression showed that BM patients with abnormal CSF appearance, $\mathrm{pH}$, and high WBCs were at higher risk of fatality compared to the reference 
Table 3 Comparative Analysis of Bacterial Culture and Gram Staining Among Bacterial Meningitis Cases

\begin{tabular}{|l|c|c|c|}
\hline \multirow{2}{*}{ Test Performed } & \multicolumn{2}{|c|}{$\begin{array}{c}\text { Bacterial Culture BM } \\
\text { Cases }\end{array}$} & \multirow{2}{*}{ Total } \\
\cline { 2 - 3 } & $\begin{array}{c}\text { Positive } \\
\text { Cases }\end{array}$ & $\begin{array}{c}\text { Negative } \\
\text { Cases }\end{array}$ & \\
\hline Gram Staining positive cases & 50 & 0 & 50 \\
Gram Staining negative cases & 271 & 573 & 844 \\
Total & 321 & 573 & 894 \\
\hline
\end{tabular}

group. The likelihood of case fatality was increased even more if the BM patients had endocarditis as an underlying disease.

\section{Sanger Sequencing and Phylogenetic Analysis}

The 16S rRNA PCR-based detection of bacteria in CSF of BM patients was confirmed the presence of $S$. pneumoniae $14.7 \%$ (130/984), S. aureus 6.9\% (61/984), N. meningitidis $5.7 \%$ (51/984), H. influenzae 2.5\% (22/984), E. coli $4.5 \%$ (40/984), and K. pneumoniae 1.9\% (17/984) (Figure 3). We submitted the sequences to GenBank, NCBI, under following accession numbers: S. aureus (MW148201.1, MW148202.1, MW148203.1, MW148204.1, MT075573.1), S. pneumoniae (MW405781, MW405782, MW405783, MW405784, MW405785, MT075569.1), H. influenzae (MZ348556, MZ348557, MZ348558, MZ348559, MZ348560) N. meningitidis (MZ318247, MZ318248, MZ318249, MZ318250, MZ318251), E. coli (MT075572, MT916768, MT916769, MT916770); K. pneumoniae (MW208813, MW208814, MW208815, MW208816, MW208817, MT102939). The phylogenetic analysis of 16S rRNA gene sequences showed the genetic relationship between our isolated strains and the reference strains (Figure 4). Isolated E. coli strains showed a cluster with two reference strains (KY655034 and KY655046) isolated from the blood cultures of Turkish patients (Figure 2). Isolated K. pneumoniae strains clustered with pathogenic K. pneumoniae strain (KT261771 and KT261773) isolated from Chinese patients. $N$. meningitidis strains showed similarity with reference strain isolated from American patient's CSF (NR_104946.1). H. influenzae strains showed similarity with Japanese (AB377164.1) and Nigerian (AM420172.1) reference strains. S. aureus strains clustered with Saudi Arabian and Pakistani reference strains isolated from hospitalized patients. The isolated S. pneumoniae strains had high similarity with Japanese (NR_114172.1) and Hong Kong (AY525795.1) strains separated from the sputum of patient. The phylogenetic tree showed the genetic and evolutionary relationship between our isolates in addition to pathogenic strains prevailing in Asia (Figure 4).

\section{Antibiotic Susceptibility Test}

We estimated antibiotic susceptibility of all bacterial isolates from BM patients (see Table 6). S. aureus isolates showed highest resistant to erythromycin $90.2 \%(55 / 61)$ and oxytetracycline $65.6 \%$ (40/61), while demonstrated high sensitivity against ciprofloxacin $96.7 \%(59 / 61)$ and levofloxacin $73.8 \%$ (45/61). S. pneumoniae isolates were $100 \%(130 / 130)$ susceptible to both levofloxacin and vancomycin. However, it showed highest resistant against amoxicillin 100\% (130/130) and rifampicin 59\% (77/130). All H. influenzae isolates were 100\% (22/22) susceptible to meropenem, ceftazidime and levofloxacin. $N$. meningitidis isolates were highly susceptible to meropenem 100\% (51/51), rifampicin 98\% (50/51), ceftriaxone $96.1 \%$ (49/51) and ciprofloxacin 92.2\% (47/51). E. coli isolates demonstrated high resistance rate against

Table 4 Incidence Rate of Isolated Bacterial Meningitis Pathogens Among Various Age Groups

\begin{tabular}{|c|c|c|c|c|c|c|c|c|}
\hline \multirow{2}{*}{$\begin{array}{l}\text { Bacteria Causing } \\
\text { Meningitis }\end{array}$} & \multicolumn{8}{|c|}{ Among Various Age Groups } \\
\hline & $\begin{array}{c}\text { I Month-3 } \\
\text { Months n(\%) }\end{array}$ & $\begin{array}{l}4 \text { Months-I } \\
\text { Year n(\%) }\end{array}$ & $\begin{array}{l}2-12 \\
\text { Years } \\
\text { n(\%) }\end{array}$ & $\begin{array}{c}13-19 \\
\text { Years } \\
n(\%)\end{array}$ & $\begin{array}{c}20-40 \\
\text { Years } \\
n(\%)\end{array}$ & $\begin{array}{c}4 I-60 \\
\text { Years } \\
n(\%)\end{array}$ & $\begin{array}{l}61-90 \\
\text { Years } \\
n(\%)\end{array}$ & Total n(\%) \\
\hline E. coli & $25(2.8 \%)$ & $12(1.3 \%)$ & $0(0 \%)$ & $0(0 \%)$ & $0(0 \%)$ & $0(0 \%)$ & $3(0.3 \%)$ & 40 (4.5\%) \\
\hline S. aureus & $12(1.3 \%)$ & $9(1.0 \%)$ & $13(1.5 \%)$ & $7(0.8 \%)$ & $6(0.7 \%)$ & $3(0.3 \%)$ & $\mathrm{II}(\mathrm{I} .2 \%)$ & 61 (6.8\%) \\
\hline K. Pneumoniae & $5(0.6 \%)$ & $7(0.8 \%)$ & $0(0 \%)$ & $0(0 \%)$ & $0(0 \%)$ & $0(0 \%)$ & $5(0.6 \%)$ & $17(1.9 \%)$ \\
\hline S. pneumoniae & 17 (1.9\%) & 27 (3.0\%) & $32(3.6 \%$ & $6(0.7 \%)$ & II (I.2\%) & 17 (1.9\%) & $20(2.2 \%)$ & $130(\mid 4.5 \%)$ \\
\hline H. influenzae & $5(0.6 \%)$ & $8(0.9 \%)$ & I (0.I\%) & $0(0 \%)$ & $0(0 \%)$ & $0(0 \%)$ & $8(0.9 \%)$ & $22(2.5 \%)$ \\
\hline N. meningitidis & 30 (3.4\%) & 17 (1.9\%) & $0(0 \%)$ & $0(0 \%)$ & $0(0 \%)$ & $0(0 \%)$ & $4(0.4 \%)$ & 51 (5.7\%) \\
\hline
\end{tabular}


Table 5 Prognostic Factors of Bacterial Meningitis Infected Patients

\begin{tabular}{|c|c|c|c|c|c|c|c|}
\hline \multirow[t]{2}{*}{ Characteristics } & \multirow[t]{2}{*}{$\begin{array}{l}\text { Total BM Cases } \\
\text { n(\%)/ Out of } 894\end{array}$} & \multirow{2}{*}{$\begin{array}{c}\text { Non-Fatal } \\
\text { BM Cases } \\
\text { n (\%) }\end{array}$} & \multirow{2}{*}{$\begin{array}{c}\text { Fatal BM } \\
\text { Cases } \\
\text { n (\%) }\end{array}$} & \multirow[t]{2}{*}{$P$ value } & \multirow[t]{2}{*}{$\begin{array}{l}\text { Odd } \\
\text { Ratio }\end{array}$} & \multicolumn{2}{|c|}{$\begin{array}{l}\text { 95\% Confidence } \\
\text { Interval }\end{array}$} \\
\hline & & & & & & Lower & Upper \\
\hline Male & 35 (61.4\%) & 159 & 27 & 0.512 & 0.634 & 0.0162 & $2.476^{\mathrm{a}}$ \\
\hline Female & $22(38.6 \%)$ & 126 & 9 & & & & \\
\hline \multicolumn{8}{|l|}{ Antimicrobial therapy before LP } \\
\hline Within $24 \mathrm{hrs}$ & $103(11.5 \%)$ & 89 & 14 & 0.909 & 1.097 & 0.225 & $5.341^{\mathrm{b}}$ \\
\hline After $24 \mathrm{hrs}$ & $218(24.4 \%)$ & 196 & 22 & & & & \\
\hline \multicolumn{8}{|l|}{ CSF pH } \\
\hline $7.32-7.5$ & $90(10.1 \%)$ & 69 & 21 & $0.01 *$ & 0.129 & 0.026 & $0.645^{c}$ \\
\hline $7.6-8$ & 231 (25.8\%) & 216 & 15 & & & & \\
\hline \multicolumn{8}{|l|}{ CSF appearance } \\
\hline Clear & $120(13.4 \%)$ & 112 & 8 & $0.00 I^{*}$ & 22.847 & 3.830 & $136.287^{d}$ \\
\hline Turbidity & $20 \mathrm{I}(22.5 \%)$ & 173 & 28 & & & & \\
\hline \multicolumn{8}{|l|}{ CSF protein level (Range) } \\
\hline $14-45 \mathrm{mg} / \mathrm{dL}$ & $13(1.5 \%)$ & 11 & 2 & 0.993 & 1.015 & 0.038 & $27.049^{e}$ \\
\hline$>$ 45mg/dL (Range; 46-2500mg/dL) & 308 (34.5\%) & 274 & 34 & & & & \\
\hline \multicolumn{8}{|l|}{ CSF glucose level (Range) } \\
\hline $45-100 \mathrm{mg} / \mathrm{dL}$ & $64(7.2 \%)$ & 58 & 6 & 0.449 & 2.205 & 0.285 & $17.058^{f}$ \\
\hline$>100 \mathrm{mg} / \mathrm{dL}$ (Range; $100-250$ mg/dL) & $9(1 \%)$ & 9 & 0 & 0.998. & 6017.95 & 6015.951 & $6021.95 I^{f}$ \\
\hline Low $\leq 45 \mathrm{mg} / \mathrm{dL}$ (Range; $0-44 \mathrm{mg} / \mathrm{dL}$ ) & $248(27.7 \%)$ & 218 & 30 & & & & \\
\hline \multicolumn{8}{|l|}{ CSF WBCs (Range) } \\
\hline 21 to 100 cells $/ \mathrm{mm}^{3}$ & $86(9.6 \%)$ & 73 & 13 & 0.117 & 4.694 & 0.678 & $32.518^{g}$ \\
\hline 101 to 2000 cells $/ \mathrm{mm}^{3}$ & $190(21.3 \%)$ & 178 & 12 & $0.00 I^{*}$ & 24.177 & 3.932 & $148.644^{g}$ \\
\hline$\geq 200 \mathrm{I}$ cells $/ \mathrm{mm}^{3}$ & $45(5 \%)$ & 34 & 11 & & & & \\
\hline \multicolumn{8}{|l|}{ Neutrophils } \\
\hline$>10-\geq 30 \%$ & $34(4 \%)$ & 25 & 9 & 0.118 & 0.184 & 0.022 & $1.535^{\mathrm{h}}$ \\
\hline$>31-\geq 60 \%$ & $101(11.1 \%)$ & 85 & 16 & 0.476 & 0.572 & 0.123 & $2.656^{\mathrm{h}}$ \\
\hline$>61-\geq 90 \%$ & $186(20.8 \%)$ & 175 & 11 & & & & \\
\hline \multicolumn{8}{|l|}{ Underlying diseases } \\
\hline Diabetes mellitus & $29(3.2 \%)$ & 23 & 6 & 0.918 & 0.841 & 0.031 & 23.017 \\
\hline Hypertension & 39 (4.4\%) & 34 & 5 & 0.944 & 1.082 & 0.119 & 9.855 \\
\hline COPD & 105 (11.7\%) & 95 & 10 & 0.337 & 1.862 & 0.524 & 6.625 \\
\hline Liver disease & 33 (3.7\%) & 28 & 5 & 0.765 & 0.735 & 0.097 & 5.544 \\
\hline Smoker & $36(4.02 \%)$ & 29 & 7 & 0.220 & 0.092 & 0.002 & 4.170 \\
\hline Severe pneumonia & I55 (I7.3\%) & 134 & 21 & 0.513 & 0.518 & 0.072 & 3.720 \\
\hline Bacteremia & $161(18 \%)$ & 139 & 22 & 0.861 & 0.853 & 0.145 & 5.028 \\
\hline Sinusitis & 91 (10.2\%) & 75 & 16 & 0.557 & 0.633 & 0.138 & 2.906 \\
\hline Endocarditis & 21 (2.3\%) & 17 & 4 & $0.05^{*}$ & 316.44 & 0.810 & 1235 \\
\hline Otitis media & 24 (2.7\%) & 21 & 3 & 0.997 & 1.010 & 0.005 & 194.55 \\
\hline
\end{tabular}

Notes: ${ }^{a}$ relative to female gender; ${ }^{b}$ relative to antimicrobial therapy not started within $24 \mathrm{hrs}$; ${ }^{\mathrm{c}}$ relative to high $\mathrm{pH}$; ${ }^{d}$ relative to turbidity of CSF; ${ }^{\mathrm{e}}$ relative to High protein

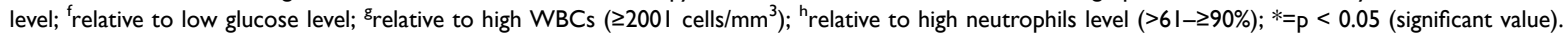




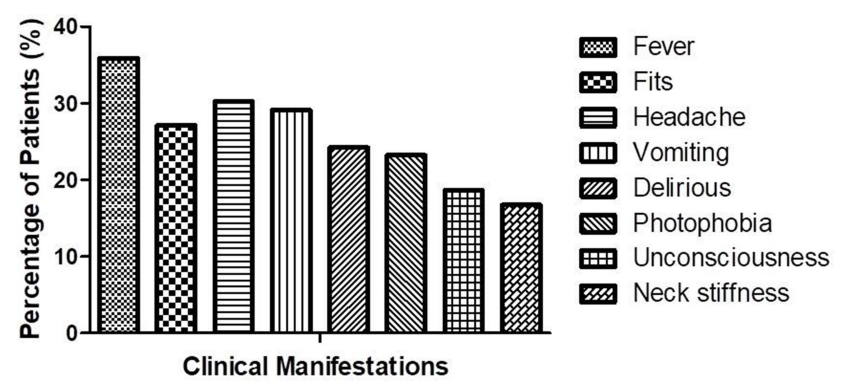

Figure 2 Clinical manifestations of bacterial meningitis patients.

amoxicillin $80 \%(32 / 40)$ and rifampicin $77.5 \%(31 / 40)$. E. coli showed highest susceptibility to meropenem $92.5 \% \quad(37 / 40)$ and amikacin $97.5 \% \quad(39 / 40)$. K. pneumoniae isolates were $100 \%$ (17/17) resistant against amoxicillin, whereas $88.2 \%(15 / 17)$ resistant to erythromycin and oxytetracycline. Highest susceptible drugs against $K$. pneumoniae were amikacin with $88.2 \%$ (15/17), levofloxacin and gentamicin 64.7\% (11/17), ceftriaxone $52.9 \%(9 / 17)$, ciprofloxacin and meropenem $58.8 \%$ (10/17), respectively (Table 6).

\section{Discussion}

In this study, we identified predominant pathogens of bacterial meningitis among the population of Quetta, Balochistan. The study showed S. pneumoniae (14.5\%), S. aureus $(6.8 \%), N$. meningitidis $(5.7 \%), H$. influenzae (2.5\%), E. coli (4.5\%) and K. pneumoniae (1.9\%) were the main causative agents. Despite variations in frequency of each pathogen, these agents remain the prime cause of BM among population. ${ }^{17-19}$ Several studies suggested that S. pneumoniae, S. aureus, E. coli and $K$. pneumoniae were commonly isolated pathogens from CSF of Pakistani children. ${ }^{20-22}$ Previously, similar studies conducted in developing and under-developed countries reported prevalence of these pathogenic strains as dominant cause of infections in CSF. ${ }^{4,20,21,23}$ There are several factors that influence the specific etiology of the disease such as immune function, status of immunization, age, genetic makeup and geographical location of patient. ${ }^{17,24}$ In this study $S$. pneumoniae and $S$. aureus were the predominant pathogens among all the age groups. S. pneumoniae globally accounts for $25.1-41.2 \%$ of BM
A

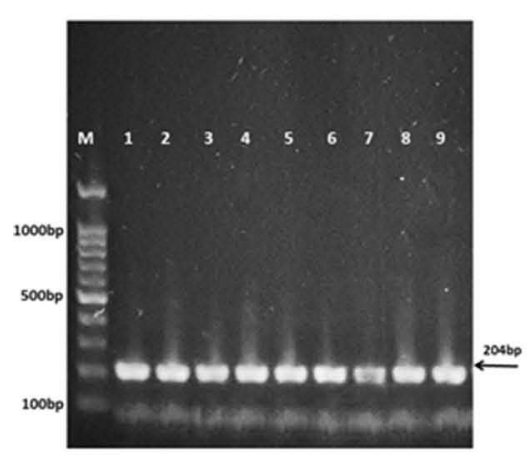

B

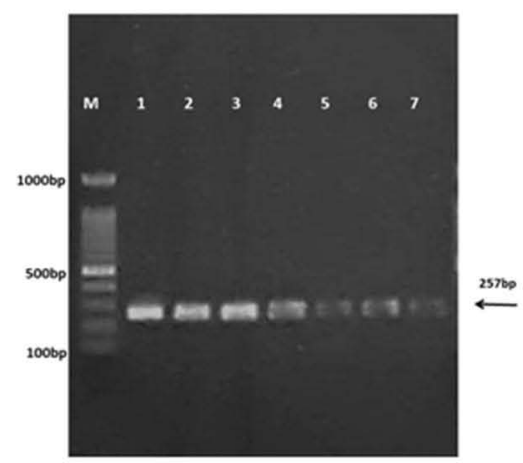

E

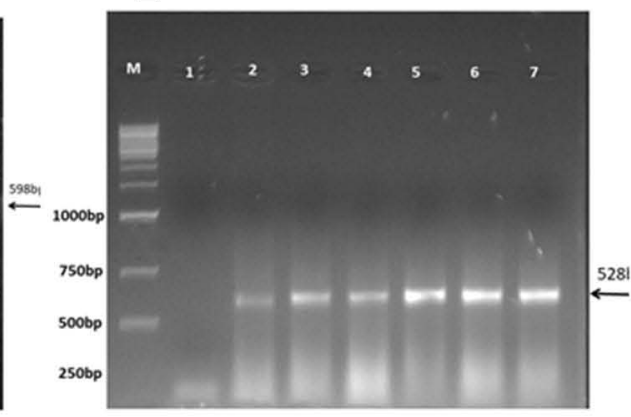

C

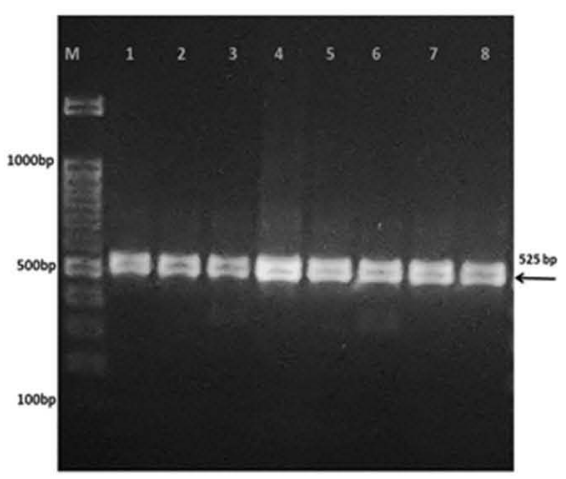

$\mathbf{F}$

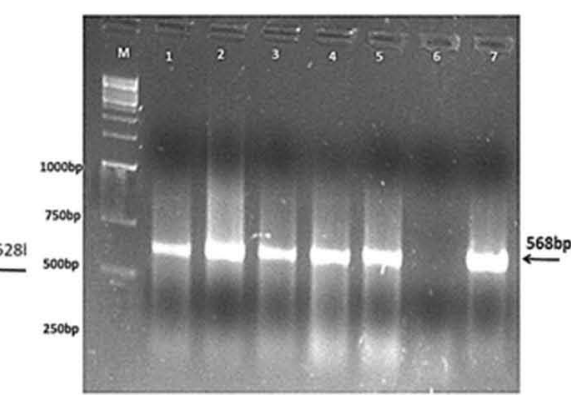

Figure 3 I6S rRNA gene PCR-based identification bacteria isolated from CSF of bacterial meningitis patients. (A) E. coli isolates with 204bp amplified fragments; (B) S. aureus solates $257 \mathrm{bp}$ amplified fragments; (C) K. pneumoniae isolates $525 \mathrm{bp}$ amplified fragments; (D) S. pneumoniae isolates $598 \mathrm{bp}$ amplified fragments; (E) $H$. influenzae isolates $528 \mathrm{bp}$ amplified fragments; (F) N. meningitidis isolates $568 \mathrm{bp}$ amplified fragments. $\mathrm{M}=$ molecular weight marker (I kb). 


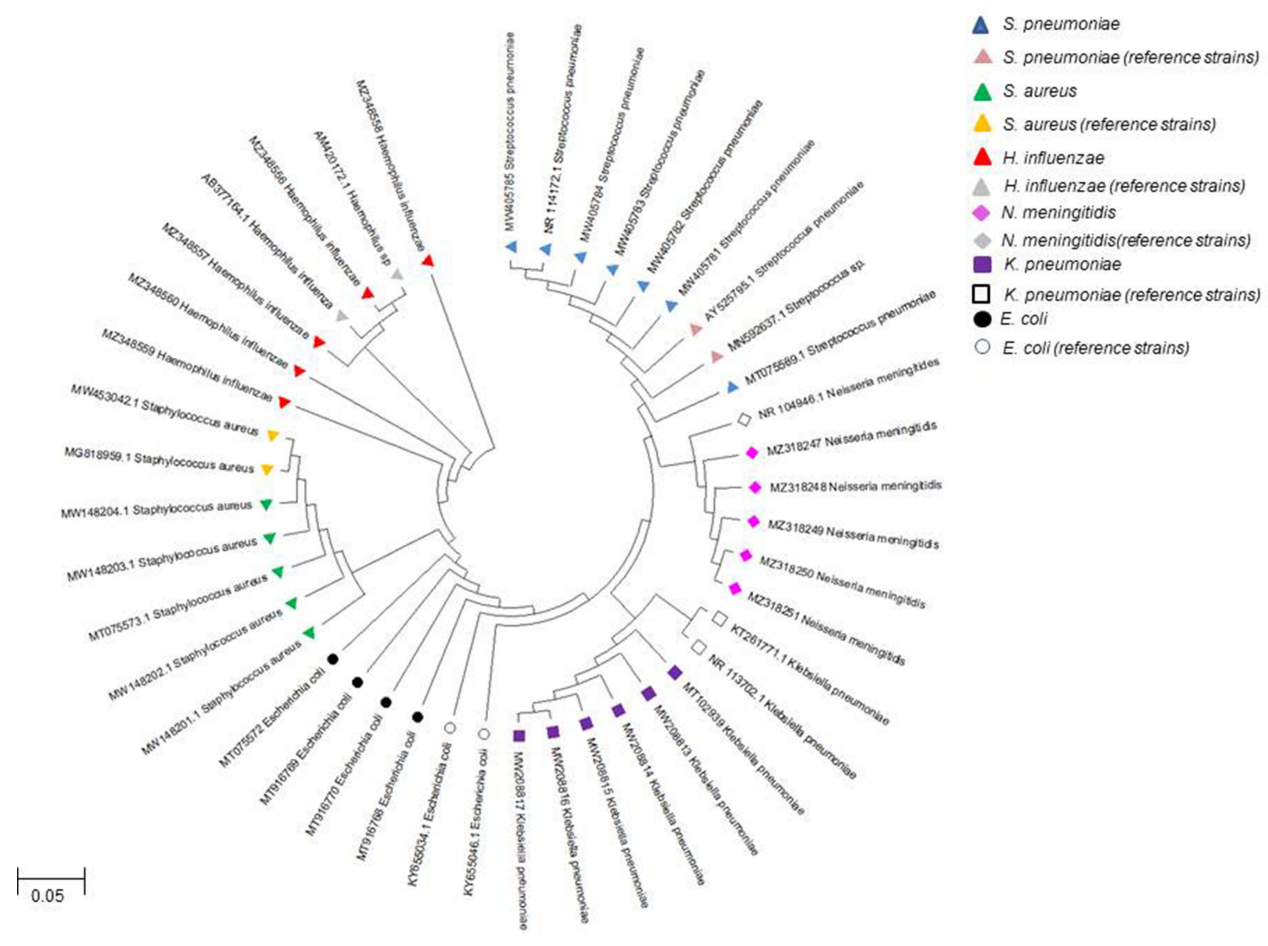

Figure 4 Phylogenetic relationship of bacterial isolates from bacterial meningitis patients with their reference strains. S. pneumoniae (MW40578I, MW405782, MW405783, MW405784, MW405785, MT075569.I); E. coli (MT075572 MT9I6768, MT9I6769, MT9I6770); K. pneumoniae (MW2088I3, MW2088I4, MW2088I5, MW2088I6, MW2088I7, MT102939); S. aureus (MWI4820I.I, MWI48202.I, MWI48203.I, MWI48204.I, MT075573.I); H. influenzae (MZ348556, MZ348557, MZ348558, MZ348559, MZ348560); N. meningitidis (MZ3I8247, MZ3I8248, MZ3I8249, MZ3I8250, MZ3I825I) isolated from BM patients of Balochistan were compared using I6S rRNA gene sequence with other reference strains using neighbor-joining phylogenetic tree. The bootstrap values were based on 1000 replicates in $M E G A X$ software.

cases across all age groups and geographic regions. ${ }^{25}$ This high incidence of $S$. pneumoniae is due to endogenous transmission from nasopharynx region to the meninges. The patients infected with pneumococcal meningitis were mostly immunocompromised, had immature nasopharynx (in children), or during dry season when the nasal cavity is particularly susceptible to damage and cracks. ${ }^{26}$

We found weak relationship between ethnicity and BM cases. We found high proportion of Pashtun ethnic group affected with BM as compared to other ethnic groups. Geographically, Balochistan shares its borders with Afghanistan and Iran and consequently receives high influx of patients from these countries. Another reason for the enormous proportion of BM cases was due to the large population of Paktoon living in rural areas of Balochistan, with inadequate health-care facilities, deplorable hygiene, a poor literacy rate, low socioeconomic status, and many clans living in congested houses. Despite inception of the Expanded Program for Immunization (EPI), the incidence of the pathogenic strains has amplified in Balochistan comparative to other regions of Pakistan. ${ }^{20-22}$ Although in many cities of Pakistan, vaccination coverage has contributed to a drastic reduction $(72 \%)$ in $H$. influenzae related meningitis cases, ${ }^{20-22,27}$ whereas in this study we found $2.5 \%$ of $H$. influenzae related BM cases. Though, EPI launched the Hib and Pneumococcal Conjugate Vaccine (PCV) programs in 2009 and 2012 respectively. The low frequency of $H$. influenzae in CSF of meningitis patients suggested that initiative of vaccination of $\mathrm{Hib}$ in the region has influenced the incidence rate and promoted herd immunity. While we found $S$. pneumoniae is the most common causative agent in BM patients. A high incidence rate of S. pneumoniae was observed among children who were unvaccinated or partially vaccinated due to low coverage of vaccination, higher urbanization among the rural population, which led to negligence and low vaccination rate among the population. ${ }^{12,28}$ According to USAID report of Pakistan, 1 in every 5 children remains unvaccinated in cities while rural areas have high rate of unvaccinated children (ie 2 out of 3 ). ${ }^{13}$ We also found significantly high percentage of children (less than one year of age) and elderly patients afflicted with $E$. coli and $K$. pneumoniae associated BM. Likewise, Jiang and coworkers found that $28.5 \%$ of $E$. coli associated cases in CSF of Chinese children. Another research demonstrated 


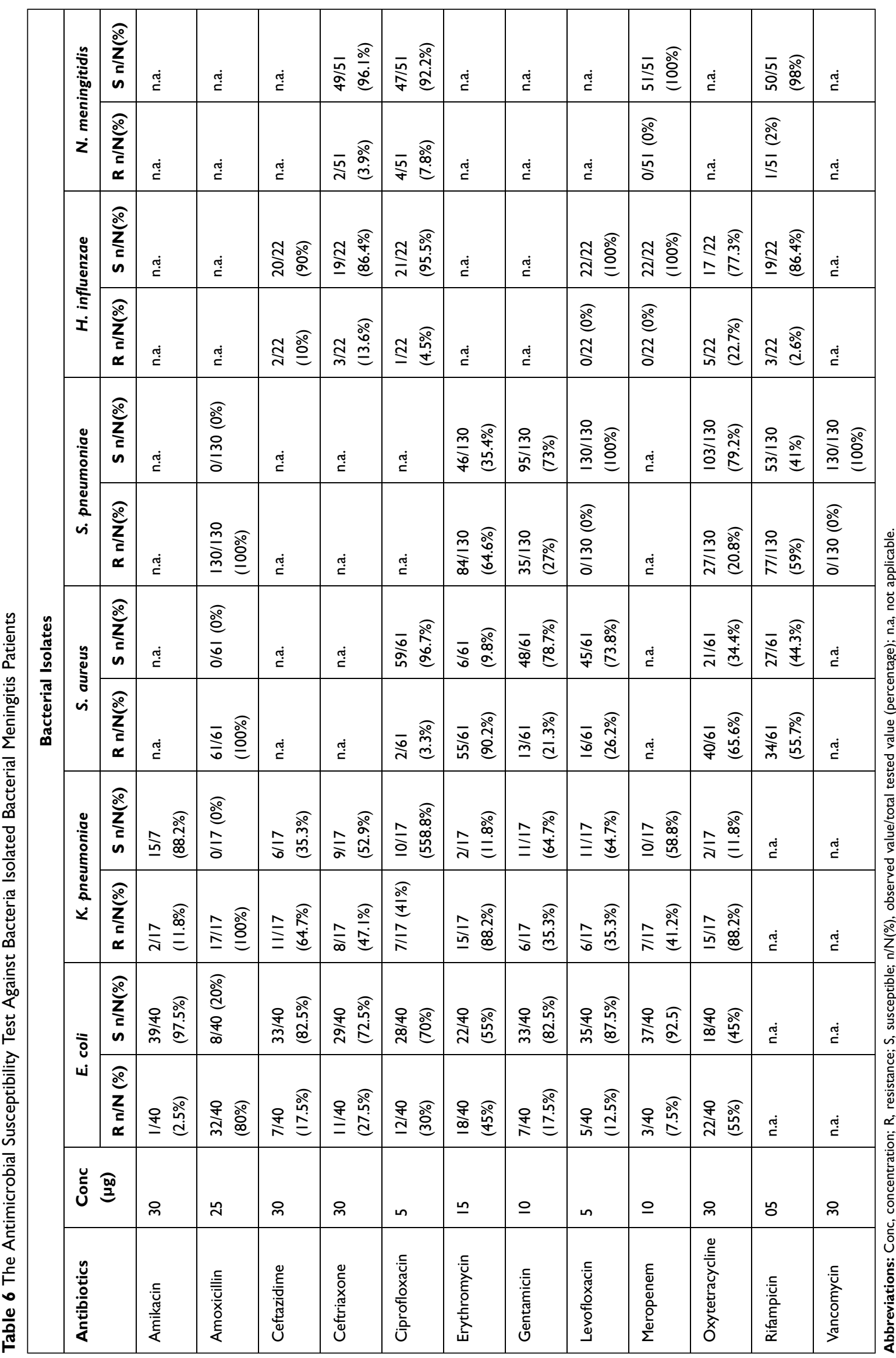


E. coli $(51.3 \%)$ and $K$. pneumoniae $(1.7 \%)$ as common causes of BM among young infants. ${ }^{4,23}$ The universally known risk factors responsible for meningitis in children, especially among infants includes: premature birth, low weight, and infection caused by pathogenic bacteria. ${ }^{29,30}$ Children less than 1 year (such as infants and neonates) are at higher risk of BM, because maternal antibodies are unable to cross the placenta (after 32 week gestation) or immune systems weaken by the neutrophils and monocyte's phagocytic activity. Moreover, bacterial infections caused by $E$. coli are responsible for meningitis and sepsis in very low birth weight babies at early stage of life. ${ }^{18,31}$ However, this disparity in our data could be due to a difference in sample size, mode of collection of CSF, or use of antibiotics prior to LP. Additionally, we found high ratio of male infants afflicted by $\mathrm{BM}$ infection as compared to female patients, this imbalance is due to the fact that female infants have strong immune system than male. ${ }^{32}$ Additionally, female's estrogen enhances level of Th1 providing stronger immune response against bacterial pathogens while testosterone diminishes the cytokines (interleukin 4 and interferon $\gamma$ ) generation in T-cells. ${ }^{32,33}$

The common signs and symptoms closely associated with BM include fever, respiratory distress or jaundice, vomiting, and lethargy. ${ }^{3}$ We also observed headache, vomiting, fits, fever, and neck stiffness as common symptom among all BM patients. However, the seizures complicate the condition of $\mathrm{BM}$ patients, especially in children. ${ }^{4}$ These clinical features along with CSF parameters and bacterial culture increase the accuracy, sensitivity, and specificity of the test by many folds in diagnosing BM. ${ }^{34}$ In $\mathrm{BM}$ patients, elevated numbers of CSF cells, proteins, and reduced levels of glucose are hallmarks of diagnosis. In this study, inflammatory changes such as protein count, glucose level, WBCs, neutrophils, and lymphocytes were prominent in CSF among $\mathrm{BM}$ patients. We found underlying chronic diseases and infections such as bacteremia, diabetes mellitus, COPD, severe pneumonia, otitis media and endocarditis as predisposing risk factors of meningitis in patients. Previous studies reported $K$. pneumoniae as a concomitant factor among patients with critical medical conditions such as meningitis, septicemia, liver abscess, pneumonia, and urinary tract inflammation leading to serious ailments and high mortalities $(33.3-48.5 \%))^{35,36}$ Several studies have reported that immuno-compromised conditions such as HIV, diabetes, and post-neurological states may contribute to the progression of bacterial meningitis. ${ }^{35}$ Similarly, endocarditis caused by $S$. pneumoniae and $S$. aureus increases the risk of BM. Another study reported that $2 \%$ of BM patients with endocarditis had higher chances $(63 \%)$ of fatal outcomes. ${ }^{37}$ In agreement with this, we found that endocarditis substantially magnifies the risk of fatality in BM patients. We observed significantly higher case fatality (11\%) rate among BM patients. According to Government of Pakistan meningitis claims 23,000 children's lives annually, ${ }^{27}$ with BM-related case fatalities around $10.1 \%$ in $2012 .^{22}$ Even though medical research has advanced rapidly with accessibility to effective therapies and potent drugs, the case fatality rates of BM (16$32 \%$ ) have exponentially accelerated in developing countries. $^{38,39}$ This rapid increase in fatality rates is a consequence of lack of basic healthcare facilities, absence of professional paramedics' staff, and the shortage of medical equipments. ${ }^{12}$ The bacterial culturing of the CSF is gold standard and routinely performed for diagnosis, yet it is a laborious and time-consuming method. The molecular detection method is suitable for BM patients with single-cell pathogens in CSF. We performed $16 \mathrm{~S}$ rRNA PCR and phylogenetic based confirmation of bacterial isolated in CSF of BM patients. The amplification and sequencing approach helped to identify taxonomy of isolates. Hence, the particular level of genetic distances and similarities within species of BM isolates and their reference strains suggested conserved close similarity via $16 \mathrm{~S}$ rRNA sequences. Therefore, early identification of the causative agents is imperative for timely therapeutic and treatment purposes.

Major challenge faced by current healthcare system is continuous rise of antibiotics resistant bacteria. We evaluated AMR and antimicrobial susceptibility (AMS) in all isolated bacterial pathogens from the CSF BM patients. The first (amoxicillin, oxytetracycline), second (levofloxacin, ciprofloxacin), third generation (ceftriaxone, ceftazidime) and other groups of antibiotics were utilized to access resistance. We found $S$. pneumoniae, E. coli and $K$. pneumoniae resistant to amoxicillin, erythromycin, and oxytetracycline. Additionally, some isolates showed resistance to 3rd generation antibiotics such as ceftazidime and ceftriaxone. We found $S$. pneumoniae significantly susceptible to vancomycin and levofloxacin. This increasing resistance to 3rd generation antibiotic is alarming as it has intensified infection among people of Balochistan. The antibiotic-resistant of $E$. coli aggravates the occurrence of infections especially in mothers and infants. Majority of infants were affected with E. coli associated 
meningitis was found $85 \%$ resistant to ampicillin. ${ }^{40}$ A meta-analysis study revealed that various regions of Pakistan, pathogenic E. coli and Klebsiella species isolated from several hospitalized patients were highly resistant to 1st generation antibiotics (penicillin 100\%, ampicillin 91\% and amoxicillin 85\%), 2nd and 3rd generation antibiotics such as cefotaxime (82\%) and cefaclor (100\%) respectively. ${ }^{41}$ This resistance is an emerging problem due to misuse and overuse of extended-spectrum cephalosporin drugs. ${ }^{10}$ The emerging resistance in $K$. pneumoniae was due to its ability to produces an enzyme that degrades the carbapenem and beta-lactam ring structures. ${ }^{44}$ We found $S$. aureus resistant to amoxicillin, erythromycin, rifampicin, oxytetracycline, and gentamicin. Similarly, in various region of Pakistan, S. aureus showed high resistance to penicillin (98\%), cefoxitin $(83 \%)$ and levofloxacin $(80 \%)$. In a recent study, Tigabu and coworkers reported high resistance $(66.80 \%)$ in S. aureus against erythromycin and amoxicillin among adult BM patients in Gondar. ${ }^{42}$ Another study conducted by Assegu Fenta and his group declared that high resistance of $S$. aureus was reported against amoxicillin $(100 \%) .{ }^{43}$ The high resistance occurs due to exposure of broad range of antibiotics, self-medication, and incomplete dosage of antibiotics, leading $S$. aureus to develop new cellular processes to evade effects of drugs. ${ }^{44}$ In this study we found all isolates of $N$. meningitidis and susceptible to all tested antibiotics whereas we found $H$. influenzae is resistance to (2-22\%) first, second, and third generation of antibiotics. While Tigabu and group reported 100\% susceptibility of $N$. meningitidis isolates against amoxicillin and erythromycin. ${ }^{42}$ The variation in antibiotic susceptibility trend among isolates might be due to late diagnostic tests and misuse of antibiotics. Hence, the increasing antibiotic resistance among various pathogens is a serious health problem. The AMR in bacteria utilizes various defense mechanisms such as restricting and pumping out drug from the bacterial cell, degrade or alter the antibiotic's structure to limit its target function. ${ }^{44}$ Despite the fact that AMR is natural process, the extensive use and misuse of antibiotics has accelerated emergence of multidrug resistance in pathogens. The AMR led antibiotics to least effective against various infectious diseases. Eventually, AMR increases economic burden due to prolonged hospital admission, expensive medical treatments and high mortality rate. ${ }^{45}$ Therefore, the emergence of antibiotic resistance in clinical isolates should be taken into account before prescribing the appropriate drug. On a broad glance, awareness is required to educate people regarding vaccination courses, personal hygiene, and selfmedication. Hence, taking these obligatory steps is vital to control the prevalence of infectious diseases and their antimicrobial resistance in the community.

\section{Conclusion}

This study for the first time investigated the incidence rate of BM in Balochistan, Pakistan. S. pneumoniae and $S$. aureus were the leading pathogens for BM and commonly found among all age group. Males were highly affected as compared to females. Extreme age groups such as infants (age range: 1 month-1 year) and adults (age range: 61 years-99 years) with low immunity were at higher risk. High case fatality rate was observed among BM patients. The increasing antibiotic resistances of causative agents are major challenge in treating patients with prudent use of empiric antibiotics. To control $\mathrm{BM}$ incidence and its fatality rate, it is important to promote AMR surveillance in all clinics and hospitals to reduce future threats. Timely detection and intensive treatment of BM may improve critical outcomes in patients. Further research is required to track the varying epidemiology of causative agents and risk factors of BM.

\section{Institutional Review Board Statement}

The research protocol was approved by the institutional research ethics committee of hospitals and institutions. This research was conducted according to Helsinki declaration, University of Balochistan (CAS/45/15-20) and hospital ethical committee's permission (E.C 18-12/ 2017).

\section{Data Sharing Statement}

Data of this study are available from corresponding author on reasonable request.

\section{Informed Consent Statement}

A written consent form was taken from patients and from parents/guardians in case of children to publish this paper. For illiterate participants, the verbal consent was obtained.

\section{Acknowledgments}

The authors gratefully acknowledge all the participants of this research. We would also like to express gratitude all doctors, physicians, and paramedic's staff for their 
contribution and support. In the end, we forward our gratitude to the director of CASVAB, University of Balochistan, Quetta; for funding and supporting this study.

\section{Author Contributions}

All authors made a significant contribution to the work reported, whether that is in the conception, study design, execution, acquisition of data, analysis and interpretation, or in all these areas; took part in drafting, revising or critically reviewing the article; gave final approval of the version to be published; have agreed on the journal to which the article has been submitted; and agree to be accountable for all aspects of the work.

\section{Disclosure}

All authors declared that they have no competing interests in this work.

\section{References}

1. Basarab M, Ihekweazu C, George R, Pebody R. Effective management in clusters of pneumococcal disease: a systematic review. Lancet Infect Dis. 2011;11(2):119-130. doi:10.1016/s1473-3099(10) 70281-4

2. Chang C-J, Chang H-W, Chang W-N, et al. Seizures complicating infantile and childhood bacterial meningitis. Pediatr Neurol. 2004;31 (3):165-171. doi:10.1016/j.pediatrneurol.2004.03.009

3. Garges HP. Neonatal meningitis: What is the correlation among cerebrospinal fluid cultures, blood cultures, and cerebrospinal fluid parameters? Pediatrics. 2006;117(4):1094-1100. doi:10.1542/peds. 2005-1132

4. Jiang $\mathrm{H}, \mathrm{Su} \mathrm{M}$, Kui L, et al. Prevalence and antibiotic resistance profiles of cerebrospinal fluid pathogens in children with acute bacterial meningitis in Yunnan province, China, 2012-2015. PLoS One. 2017;12(6):e0180161. doi:10.1371/journal.pone.0180161

5. Okike IO, Ribeiro S, Ramsay ME, Heath PT, Sharland M, Ladhani SN. Trends in bacterial, mycobacterial, and fungal meningitis in England and Wales 2004-11: an observational study. Lancet Infect Dis. 2014;14(4):301-307. doi:10.1016/s1473-3099(13)70332-3

6. Kim KS. Acute bacterial meningitis in infants and children. Lancet Infect Dis. 2010;10(1):32-42. doi:10.1016/S1473-3099(09)70306-8

7. Paireau J, Chen A, Broutin H, Grenfell B, Basta NE. Seasonal dynamics of bacterial meningitis: a time-series analysis. Lancet Glob Health. 2016;4(6):e370-e377. doi:10.1016/S2214-109X(16) 30064-X

8. Rosenstein NE, Perkins BA, Stephens DS, Popovic T, Hughes JM. Meningococcal disease. New Engl J Med. 2001;344(18):1378-1388. doi:10.1056/NEJM200105033441807

9. Kim KS. Neonatal bacterial meningitis. NeoReviews. 2015;16(9): e535-e543. doi:10.1542/neo.16-9-e535

10. Michael CA, Dominey-Howes D, Labbate M. The antimicrobial resistance crisis: causes, consequences, and management. Front Public Health. 2014;2:145. doi:10.3389/fpubh.2014.00145

11. Foxman B. Molecular epidemiology: focus on infection. $\mathrm{Am}$ J Epidemiol. 2001;153(12):1135-1141. doi:10.1093/aje/153.12.1135

12. Umer MF, Zofeen S, Hu W, Qi X, Zhuang G. Spatiotemporal clustering analysis of Expanded Program on Immunization (EPI) vaccination coverage in Pakistan. Sci Rep. 2020;10(1):1-11. doi:10.1038/ s41598-020-67839-0
13. USAID Pakistan. Childhood immunization in Pakistan; 2012.

14. Pruesse E, Peplies J, Glöckner FO. SINA: accurate high-throughput multiple sequence alignment of ribosomal RNA genes. Bioinformatics. 2012;28(14):1823-1829. doi:10.1093/bioinformatics/bts252

15. Kumar S, Stecher G, Li M, Knyaz C, Tamura K. MEGA X: molecular Evolutionary Genetics Analysis across Computing Platforms. Mol Biol Evol. 2018;35(6):1547-1549. doi:10.1093/molbev/msy096

16. CLSI. Performance Standards for Antimicrobial Susceptibility Testing. 30th ed. 2020. CLSI supplement M100-ED30

17. Brouwer MC, Tunkel AR, van de Beek D. Epidemiology, diagnosis, and antimicrobial treatment of acute bacterial meningitis. Clin Microbiol Rev. 2010;23(3):467-492. doi:10.1128/cmr.00070-09

18. Davis LE. Acute bacterial meningitis. Continuum. 2018;24 (5):1264-1283. doi:10.1212/con.0000000000000660

19. Thigpen MC, Whitney CG, Messonnier NE, et al. Bacterial meningitis in the United States, 1998-2007. N Engl J Med. 2011;364 (21):2016-2025. doi:10.1056/nejmoa1005384

20. Taj A, Jamil N. Detection of meningococcal meningitis in cerebrospinal fluid of patients with neurological disorders in government hospitals of Karachi. J Pak Med Assoc. 2016;66(11):1418-1421.

21. Zaidi Anita KM, Khan H, Lasi R, Mahesar W. Surveillance of pneumococcal meningitis among children in Sindh, Southern Pakistan. Clin Infect Dis. 2009;48(s2):S129-S135. doi:10.1086/596491

22. Bari A, Zeeshan F, Zafar A, Ejaz H, Iftikhar A, Rathore AW. Childhood acute bacterial meningitis: clinical spectrum, bacteriological profile and outcome. J Coll Physicians Surg Pak. 2016;26 (10):822-826.

23. Nhantumbo AA, Cantarelli VV, Caireão J, et al. Frequency of pathogenic paediatric bacterial meningitis in Mozambique: the critical role of multiplex real-time polymerase chain reaction to estimate the burden of disease. PLoS One. 2015;10(9):e0138249. doi:10.1371/ journal.pone. 0138249

24. Heckenberg SGB, Brouwer MC, van de Beek D. Bacterial meningitis. Handb Clin Neurol. 2014;121:1361-1375. doi:10.1016/ B978-0-7020-4088-7.00093-6

25. Oordt-Speets AM, Bolijn R, van Hoorn RC, Bhavsar A, Kyaw MH. Global etiology of bacterial meningitis: a systematic review and meta-analysis. PLoS One. 2018;13(6):e0198772. doi:10.1371/journal.pone. 0198772

26. Gervaix A, Taguebue J, Bescher BN, et al. Bacterial meningitis and pneumococcal serotype distribution in children in Cameroon. Pediatr Infect Dis J. 2012;31:1084-1087. doi:10.1097/inf.0b013e318260552d

27. Government of Pakistan. Expanded Programs on Immunization; 2018. Available from: http://www.epi.gov.pk/vaccine-preventablediseases/meningitis. Accessed November 26, 2021.

28. Pakistan Bureau of Statistics. Labour force statistics (2014-2015); 2015.

29. Sáez-Llorens X, McCracken GH. Bacterial meningitis in children. Lancet. 2003;361(9375):2139-2148. doi:10.1016/s0140-6736(03) 13693-8

30. Osrin D, Vergnano S, Costello A. Serious bacterial infections in newborn infants in developing countries. Curr Opin Infect Dis. 2004;17(3):217-224. doi:10.1097/00001432-200406000-00008

31. Ku LC, Boggess KA, Cohen-Wolkowiez M. Bacterial meningitis in infants. Clin Perinatol. 2015;42(1):29-45. doi:10.1016/j.clp.2014.10.004

32. Fish EN. The X-files in immunity: sex-based differences predispose immune responses. Nat Rev Immunol. 2008;8(9):737-744. doi:10. 1038/nri2394

33. Araneo B, Dowell T, Diegel M, Daynes R. Dihydrotestosterone exerts a depressive influence on the production of interleukin-4 (IL-4), IL-5, and gamma-interferon, but not IL-2 by activated murine T cells. Blood. 1991;78(3):688-699. doi:10.1182/blood.v78.3.688. bloodjournal 783688

34. Nigrovic LE, Kuppermann N, Macias CG, et al. Clinical prediction rule for identifying children with cerebrospinal fluid pleocytosis at very low risk of bacterial meningitis. JAMA. 2007;297(1):52-60. doi:10.1001/jama.297.1.52 
35. Borer A, Saidel-Odes L, Riesenberg K, et al. Attributable mortality rate for carbapenem-resistant Klebsiella pneumoniae bacteremia. Infect Control Hosp Epidemiol. 2009;30(10):972-976. doi:10.1086/605922

36. Lu C-H, Chang W-N, Chang H-W. Adult bacterial meningitis in southern Taiwan: epidemiologic trend and prognostic factors. J Neurol Sci. 2000;182(1):36-44. doi:10.1016/s0022-510x(00)00445-7

37. Lucas MJ, Brouwer MC, van der Ende A, van de Beek D. Endocarditis in adults with bacterial meningitis. Circulation. 2013;127(20):2056-2062. doi:10.1161/circulationaha.113.001545

38. Moat A, Jarousha A, Al Afifi A. Epidemiology and risk factors associated with developing bacterial meningitis among children in Gaza Strip. Iranian J Public Health. 2014;43(9):1176-1183.

39. Nickerson JW, Attaran A, Westerberg BD, Curtis S, Overton S, Mayer P. Fatal bacterial meningitis possibly associated with substandard ceftriaxone - Uganda, 2013. MMWR Morb Mortal Wkly Rep. 2016;64(50-51):1375-1377. doi:10.15585/mmwr.mm6450a2

40. Stoll BJ, Hansen N, Fanaroff AA, et al. Changes in pathogens causing early-onset sepsis in very-low-birth-weight infants. $N$ Engl $J$ Med. 2002;347(4):240-247. doi:10.1056/nejmoa012657

41. Bilal H, Khan MN, Rehman T, Hameed MF, Yang X. Antibiotic resistance in Pakistan: a systematic review of past decade. $B M C$ Infect Dis. 2021;21(1). doi:10.1186/s12879-021-05906-1

42. Tigabu A, Jember A, Nega T, et al. Bacterial meningitis among adult patients at University of Gondar comprehensive specialized referral hospital. Infect Drug Resist. 2021;14:565-574. doi:10.2147/idr. s296792
43. Assegu Fenta D, Lemma K, Tadele H, Tadesse BT, Derese B. Antimicrobial sensitivity profile and bacterial isolates among suspected pyogenic meningitis patients attending at Hawassa University Hospital: cross-sectional study. BMC Microbiol. 2020;20 (1):125. doi:10.1186/s12866-020-01808-5

44. CDC. How Antibiotic Resistance Happens. Centers for Disease Control and Prevention; March 20, 2019. Available from: https:// www.cdc.gov/drugresistance/about/how-resistance-happens.html. Accessed November 26, 2021.

45. World Health Organization. Antibiotic resistance. World Health Organization; July 31, 2020. Available from: https://www.who. int/news-room/fact-sheets/detail/antibiotic-resistance. Accessed November 26, 2021.

46. Wang Y, Guo G, Wang H, et al. Comparative study of bacteriological culture and real-time fluorescence quantitative PCR (RT-PCR) and multiplex PCR-based reverse line blot (mPCR/ RLB) hybridization assay in the diagnosis of bacterial neonatal meningitis. BMC Pediatr. 2014;14(1):224. doi:10.1186/1471-243114-224

47. Johnson EJ, Zemanick ET, Accurso FJ, Wagner BD, Robertson CE, Harris JK. Molecular identification of Staphylococcus aureus in airway samples from children with cystic fibrosis. PLoS One. 2016;11(1):e0147643. doi:10.1371/journal. pone. 0147643
Infection and Drug Resistance

\section{Publish your work in this journal}

Infection and Drug Resistance is an international, peer-reviewed openaccess journal that focuses on the optimal treatment of infection (bacterial, fungal and viral) and the development and institution of preventive strategies to minimize the development and spread of resistance. The journal is specifically concerned with the epidemiology of

\section{Dovepress}

antibiotic resistance and the mechanisms of resistance development and diffusion in both hospitals and the community. The manuscript management system is completely online and includes a very quick and fair peerreview system, which is all easy to use. Visit http://www.dovepress.com/ testimonials.php to read real quotes from published authors. 